\title{
Hamiltonian cohomological derivation of four-dimensional nonlinear gauge theories
}

\author{
C. Bizdadea, E. M. Cioroianu ${ }^{\dagger}$ S. O. Saliu ${ }^{\ddagger}$ \\ Faculty of Physics, University of Craiova \\ 13 A. I. Cuza Str., Craiova RO-1100, Romania
}

December 4, 2018

\begin{abstract}
Consistent couplings among a set of scalar fields, two types of oneforms and a system of two-forms are investigated in the light of the Hamiltonian BRST cohomology, giving a four-dimensional nonlinear gauge theory. The emerging interactions deform the first-class constraints, the Hamiltonian gauge algebra, as well as the reducibility relations.
\end{abstract}

PACS number: 11.10.Ef

\section{Introduction}

The Hamiltonian version of the BRST symmetry [1], [2] imposed itself as an appropriate setting for analysing various topics in gauge theories, such as its implementation in quantum mechanics [1] (Chapter 14), or the appropriate correlation between the BRST symmetry itself and canonical quantization methods [3]. Meanwhile, the cohomological development of the BRST method was proved to be a useful tool for approaching some less known aspects, like the Hamiltonian analysis of anomalies [4], the precise relation

*e-mail address: bizdadea@central.ucv.ro

$\dagger$ †-mail address: manache@central.ucv.ro

†e-mail address: osaliu@central.ucv.ro 
between the local Lagrangian and Hamiltonian BRST cohomologies [5], and, recently, the problem of obtaining consistent Hamiltonian interactions in gauge theories by means of the deformation theory [6].

In this paper we investigate the consistent Hamiltonian interactions that can be added among a set of scalar fields, two types of one-forms and a system of two-forms in four dimensions, described in the free limit by an abelian BF theory [7], which result in a four-dimensional nonlinear gauge theory. This paper extends our results related to the two-dimensional case 80 . It is known that nonlinear gauge theories in two dimensions [9] are already important as they are related to pure two-dimensional gravitation theory [10]. Indeed, when the nonlinear algebra is the Lorentz-covariant extension of the Poincaré algebra, one recovers nothing but the Yang-Mills-like formulation of $\mathbf{R}^{2}$ gravity with dynamical torsion, the so-called 'dilaton' gravity [11]. In this light, it appears quite clear that the derivation of nonlinear gauge theories in dimensions higher than two might bring significant contribution to the evolvement of a conceptual mechanism for the study of quantum gravity in higher dimensions from the perspective of gauge theories.

Our strategy is as follows. Initially, we derive the Hamiltonian BRST symmetry of the abelian BF theory in four dimensions, which splits as the sum between the Koszul-Tate differential and the exterior derivative along the gauge orbits. Next, we solve the main equations governing the Hamiltonian deformation procedure on behalf of the BRST cohomology of the free theory. As a consequence, we find the BRST charge and BRST-invariant Hamiltonian of the deformed model. With the help of these deformed quantities, we identify the interacting gauge theory by analysing the resulting first-class constraints, first-class Hamiltonian and gauge algebra.

Our paper is organized in seven sections. Section 2 briefly reviews the problem of constructing consistent Hamiltonian interactions in the framework of the BRST formalism. In Section 3 we derive the BRST symmetry of the free model. In Sections 4 and 5 we compute the deformed BRST charge, respectively, the deformed BRST-invariant Hamiltonian. Section 6 focuses on the identification of the interacting model. Section 7 ends the paper with the main conclusions. 


\section{Main equations of the Hamiltonian defor- mation procedure}

It has been shown in [6] that the problem of constructing consistent Hamiltonian interactions in theories with first-class constraints can be reformulated as a deformation problem of the BRST charge $\Omega_{0}$ and of the BRST-invariant Hamiltonian $H_{0 \mathrm{~B}}$ of a given "free" first-class theory. If the interactions can be consistently constructed, then the "free" BRST charge can be deformed as

$$
\begin{aligned}
& \Omega_{0} \rightarrow \Omega=\Omega_{0}+g \int d^{D-1} x \omega_{1}+g^{2} \int d^{D-1} x \omega_{2}+O\left(g^{3}\right) \\
= & \Omega_{0}+g \Omega_{1}+g^{2} \Omega_{2}+O\left(g^{3}\right),
\end{aligned}
$$

where $\Omega$ should satisfy the equation

$$
[\Omega, \Omega]=0 .
$$

Equation (2) splits accordingly the deformation parameter $g$ as

$$
\begin{gathered}
{\left[\Omega_{0}, \Omega_{0}\right]=0,} \\
2\left[\Omega_{0}, \Omega_{1}\right]=0, \\
2\left[\Omega_{0}, \Omega_{2}\right]+\left[\Omega_{1}, \Omega_{1}\right]=0,
\end{gathered}
$$

Obviously, equation (3) is automatically satisfied. From the remaining equations we deduce the pieces $\left(\Omega_{k}\right)_{k>0}$ on account of the "free" BRST differential. With the deformed BRST charge at hand, we then deform the BRSTinvariant Hamiltonian of the "free" theory

$$
\begin{aligned}
& H_{0 \mathrm{~B}} \rightarrow H_{\mathrm{B}}=H_{0 \mathrm{~B}}+g \int d^{D-1} x h_{1}+g^{2} \int d^{D-1} x h_{2}+O\left(g^{3}\right) \\
= & H_{0 \mathrm{~B}}+g H_{1}+g^{2} H_{2}+O\left(g^{3}\right),
\end{aligned}
$$

and require that

$$
\left[H_{\mathrm{B}}, \Omega\right]=0 .
$$

Like in the previous case, equation (7) can be decomposed accordingly the deformation parameter like

$$
\left[H_{0 \mathrm{~B}}, \Omega_{0}\right]=0,
$$




$$
\begin{gathered}
{\left[H_{0 \mathrm{~B}}, \Omega_{1}\right]+\left[H_{1}, \Omega_{0}\right]=0,} \\
{\left[H_{0 \mathrm{~B}}, \Omega_{2}\right]+\left[H_{1}, \Omega_{1}\right]+\left[H_{2}, \Omega_{0}\right]=0,}
\end{gathered}
$$

Clearly, equation (8) is again fulfilled, while from the other equations one can determine the components $\left(H_{k}\right)_{k>0}$ by relying on the BRST symmetry of the "free" model. Once the deformations are computed, special attention should be paid to the elimination of non-locality, as well as of triviality of the resulting deformations.

\section{BRST symmetry of the free theory}

We begin with a free model that describes an abelian four-dimensional BF theory involving a set of scalar fields, two types of one-forms and a collection of two-forms

$$
S_{0}\left[A_{\mu}^{a}, H_{\mu}^{a}, \varphi_{a}, B_{a}^{\mu \nu}\right]=\int d^{4} x\left(H_{\mu}^{a} \partial^{\mu} \varphi_{a}+\frac{1}{2} B_{a}^{\mu \nu} \partial_{[\mu} A_{\nu]}^{a}\right),
$$

where the notation $[\mu \nu]$ signifies antisymmetry with respect to the indices between brackets. The above action is invariant under the gauge transformations

$$
\delta_{\epsilon} A_{\mu}^{a}=\partial_{\mu} \epsilon^{a}, \delta_{\epsilon} H_{\mu}^{a}=\partial^{\nu} \epsilon_{\mu \nu}^{a}, \delta_{\epsilon} \varphi_{a}=0, \delta_{\epsilon} B_{a}^{\mu \nu}=\partial_{\rho} \epsilon_{a}^{\mu \nu \rho},
$$

which are off-shell second-stage reducible, where the gauge parameters $\epsilon^{a}$, $\epsilon_{\mu \nu}^{a}$ and $\epsilon_{a}^{\mu \nu \rho}$ are bosonic, the last two sets being completely antisymmetric.

After the elimination of the second-class constraints (the co-ordinates of the reduced phase-space are $\left.z^{A}=\left(\pi_{a}^{0}, A_{\mu}^{a}, B_{a}^{\mu \nu}, p_{a}^{i}, H_{\mu}^{a}, \pi_{i j}^{a}, \varphi_{a}\right)\right)$, we are left with a system subject only to the first-class constraints

$$
\begin{gathered}
G_{a}^{(1)} \equiv \pi_{a}^{0} \approx 0, G_{a}^{(2)} \equiv-\partial_{i} B_{a}^{0 i} \approx 0, \\
G_{i j}^{(1) a} \equiv \pi_{i j}^{a} \approx 0, G_{i j}^{(2) a} \equiv-\frac{1}{2} \partial_{[i} A_{j]}^{a} \approx 0, \\
\gamma_{a}^{(1) i} \equiv p_{a}^{i} \approx 0, \gamma_{a}^{(2) i} \equiv-\partial^{i} \varphi_{a} \approx 0,
\end{gathered}
$$

and displaying the first-class Hamiltonian

$$
H_{0}=\int d^{3} x\left(H_{i}^{a} \gamma_{a}^{(2) i}+B_{a}^{i j} G_{i j}^{(2) a}+A_{0}^{a} G_{a}^{(2)}\right),
$$


in terms of the non-vanishing fundamental Dirac brackets

$$
\begin{gathered}
{\left[\pi_{a}^{0}(t, \mathbf{x}), A_{0}^{b}(t, \mathbf{y})\right]=-\delta_{a}^{b} \delta^{3}(\mathbf{x}-\mathbf{y})} \\
{\left[B_{a}^{0 i}(t, \mathbf{x}), A_{j}^{b}(t, \mathbf{y})\right]=-\delta_{j}^{i} \delta_{a}^{b} \delta^{3}(\mathbf{x}-\mathbf{y}),} \\
{\left[H_{0}^{a}(t, \mathbf{x}), \varphi_{b}(t, \mathbf{y})\right]=-\delta_{b}^{a} \delta^{3}(\mathbf{x}-\mathbf{y}),} \\
{\left[\pi_{i j}^{a}(t, \mathbf{x}), B_{b}^{k l}(t, \mathbf{y})\right]=-\frac{1}{2} \delta_{b}^{a} \delta_{i}^{[k} \delta_{j}^{l]} \delta^{3}(\mathbf{x}-\mathbf{y}),} \\
{\left[p_{a}^{i}(t, \mathbf{x}), H_{j}^{b}(t, \mathbf{y})\right]=-\delta_{j}^{i} \delta_{a}^{b} \delta^{3}(\mathbf{x}-\mathbf{y}) .}
\end{gathered}
$$

The above constraints are abelian, while the remaining gauge algebra relations are expressed by

$$
\begin{gathered}
{\left[H_{0}, G_{a}^{(1)}\right]=G_{a}^{(2)},\left[H_{0}, G_{a}^{(2)}\right]=0} \\
{\left[H_{0}, G_{i j}^{(1) a}\right]=G_{i j}^{(2) a},\left[H_{0}, G_{i j}^{(2) a}\right]=0} \\
{\left[H_{0}, \gamma_{a}^{(1) i}\right]=\gamma_{a}^{(2) i},\left[H_{0}, \gamma_{a}^{(2) i}\right]=0}
\end{gathered}
$$

The constraint functions $G_{i j}^{(2) a}$ are first-stage reducible, with the reducibility functions given by

$$
Z_{a k l m}^{b i j}=\frac{1}{2} \delta_{a}^{b} \partial_{[k} \delta_{l}^{i} \delta_{m]}^{j},
$$

while the constraint functions $\gamma_{a}^{(2) i}$ are second-stage reducible, where the first-, respectively, second-stage reducibility functions read as

$$
Z_{b i}^{a j k}=\delta_{b}^{a} \partial^{[j} \delta_{i}^{k]}, Z_{c j k}^{b l m n}=\frac{1}{2} \delta_{c}^{b} \partial^{[l} \delta_{j}^{m} \delta_{k}^{n]} .
$$

We mention that all the reducibility relations hold off-shell.

The Hamiltonian BRST formalism requires the introduction of the ghosts

$$
\begin{gathered}
\eta^{a_{0}}=\left(\eta^{(1) a}, \eta^{(2) a}, \eta_{a}^{(1) i j}, \eta_{a}^{(2) i j}, C_{i}^{(1) a}, C_{i}^{(2) a}\right), \\
\eta^{a_{1}}=\left(C_{i j}^{a}, \eta_{a}^{i j k}\right), \eta^{a_{2}}=\left(C_{i j k}^{a}\right),
\end{gathered}
$$

together with their conjugated antighosts

$$
\mathcal{P}_{a_{0}}=\left(\mathcal{P}_{a}^{(1)}, \mathcal{P}_{a}^{(2)}, \mathcal{P}_{i j}^{(1) a}, \mathcal{P}_{i j}^{(2) a}, P_{a}^{(1) i}, P_{a}^{(2) i}\right)
$$




$$
\mathcal{P}_{a_{1}}=\left(P_{a}^{i j}, \mathcal{P}_{i j k}^{a}\right), \mathcal{P}_{a_{2}}=\left(P_{a}^{i j k}\right) .
$$

In (27 28), the fields $\eta^{a_{0}}$ and $\eta^{a_{2}}$ are fermionic, with the ghost number equal to one, respectively, to three, while $\eta^{a_{1}}$ are bosonic, of ghost number two. The ghost number is defined in the usual manner as the difference between the pure ghost number (pgh) and the antighost number (antigh), where

$$
\begin{gathered}
\operatorname{pgh}\left(z^{A}\right)=0, \operatorname{pgh}\left(\eta^{a_{0}}\right)=1, \operatorname{pgh}\left(\mathcal{P}_{a_{0}}\right)=0, \\
\operatorname{antigh}\left(z^{A}\right)=0, \operatorname{antigh}\left(\eta^{a_{0}}\right)=0, \operatorname{antigh}\left(\mathcal{P}_{a_{0}}\right)=1, \\
\operatorname{pgh}\left(\eta^{a_{1}}\right)=2, \operatorname{pgh}\left(\mathcal{P}_{a_{1}}\right)=0, \\
\operatorname{antigh}\left(\eta^{a_{1}}\right)=0, \operatorname{antigh}\left(\mathcal{P}_{a_{1}}\right)=2, \\
\operatorname{pgh}\left(\eta^{a_{2}}\right)=3, \operatorname{pgh}\left(\mathcal{P}_{a_{2}}\right)=0, \\
\operatorname{antigh}\left(\eta^{a_{2}}\right)=0, \operatorname{antigh}\left(\mathcal{P}_{a_{2}}\right)=3 .
\end{gathered}
$$

The BRST charge of the free model under discussion takes the form

$$
\begin{aligned}
& \Omega_{0}=\int d^{3} x\left(\eta^{(1) a} G_{a}^{(1)}+\eta^{(2) a} G_{a}^{(2)}+\eta_{a}^{(1) i j} G_{i j}^{(1) a}+\eta_{a}^{(2) i j} G_{i j}^{(2) a}+C_{i}^{(1) a} \gamma_{a}^{(1) i}\right. \\
& \left.+C_{i}^{(2) a} \gamma_{a}^{(2) i}+C_{i j}^{a} \partial^{[i} P_{a}^{(2) j]}+\eta_{a}^{i j k} \partial_{[i} \mathcal{P}_{j k]}^{(2) a}+C_{i j k}^{a} \partial^{[i} P_{a}^{j k]}\right)
\end{aligned}
$$

while the corresponding BRST-invariant Hamiltonian is

$$
H_{0 \mathrm{~B}}=H_{0}+\int d^{3} x\left(\eta^{(1) a} \mathcal{P}_{a}^{(2)}+C_{i}^{(1) a} P_{a}^{(2) i}+\eta_{a}^{(1) i j} \mathcal{P}_{i j}^{(2) a}\right) .
$$

The BRST symmetry of the free theory, $s \bullet=\left[\bullet, \Omega_{0}\right]$, splits as

$$
s=\delta+\gamma,
$$

where $\delta$ denotes the Koszul-Tate differential $(\operatorname{antigh}(\delta)=-1, \operatorname{pgh}(\delta)=$ $0)$, and $\gamma$ represents the exterior longitudinal derivative $(\operatorname{antigh}(\gamma)=0$, $\operatorname{pgh}(\gamma)=1$ ). These two operators act on the variables from the BRST complex like

$$
\begin{gathered}
\delta z^{A}=0, \delta \eta^{a_{0}}=0, \delta \eta^{a_{1}}=0, \delta \eta^{a_{2}}=0 \\
\delta \mathcal{P}_{a}^{(1)}=-\pi_{a}^{0}, \delta \mathcal{P}_{a}^{(2)}=\partial_{i} B_{a}^{0 i}, \delta P_{a}^{(1) i}=-p_{a}^{i}, \delta P_{a}^{(2) i}=\partial^{i} \varphi_{a} \\
\delta \mathcal{P}_{i j}^{(1) a}=-\pi_{i j}^{a}, \delta \mathcal{P}_{i j}^{(2) a}=\frac{1}{2} \partial_{[i} A_{j]}^{a}, \delta P_{a}^{i j}=-\partial^{[i} P_{a}^{(2) j]}
\end{gathered}
$$




$$
\begin{gathered}
\delta \mathcal{P}_{i j k}^{a}=-\partial_{[i} \mathcal{P}_{j k]}^{(2) a}, \delta P_{a}^{i j k}=-\partial^{[i} P_{a}^{j k]}, \\
\gamma A_{i}^{a}=\partial_{i} \eta^{(2) a}, \gamma A_{0}^{a}=\eta^{(1) a}, \gamma \varphi_{a}=0, \gamma \pi_{a}^{0}=0, \gamma p_{a}^{i}=0, \gamma \pi_{i j}^{a}=0 \\
\gamma B_{a}^{0 i}=\partial_{j} \eta_{a}^{(2) i j}, \gamma B_{a}^{i j}=\eta_{a}^{(1) i j}, \gamma H_{i}^{a}=C_{i}^{(1) a}, \gamma H_{0}^{a}=-\partial^{i} C_{i}^{(2) a} \\
\gamma \eta^{(1) a}=\gamma \eta^{(2) a}=\gamma C_{i}^{(1) a}=\gamma \eta_{a}^{(1) i j}=0 \\
\gamma C_{i}^{(2) a}=-2 \partial^{j} C_{i j}^{a}, \gamma C_{i j}^{a}=-3 \partial^{k} C_{i j k}^{a}, \gamma \eta_{a}^{(2) i j}=3 \partial_{k} \eta_{a}^{i j k} \\
\gamma \eta_{a}^{i j k}=0, \gamma C_{i j k}^{a}=0, \gamma \mathcal{P}_{a_{0}}=0, \gamma \mathcal{P}_{a_{1}}=0, \gamma \mathcal{P}_{a_{2}}=0 .
\end{gathered}
$$

The last formulas will be employed in the next section for the deformation of the free theory.

\section{Deformed BRST charge}

In this section we approach the equations that govern the deformation of the BRST charge by relying on cohomological techniques. As a result, we find that only the first-order deformation is non-trivial, while its consistency reveals the Jacobi identity for a nonlinear algebra.

\subsection{First-order deformation}

Initially, we solve the equation (4), which is responsible for the first-order deformation of the BRST charge. It takes the local form

$$
s \omega_{1}=\partial_{i} j^{i}
$$

for some local $j^{i}$. In order to investigate this equation, we develop $\omega_{1}$ according to the antighost number

$$
\omega_{1}=\stackrel{(0)}{\omega}_{1}+\stackrel{(1)}{\omega}_{1}+\cdots+\stackrel{(J)}{\omega}_{1} \text {, antigh }\left(\stackrel{(I)}{\omega}_{1}\right)=I,
$$

where the last term in the last formula can be assumed to be annihilated by $\gamma$, $\gamma \stackrel{(J)}{\omega}_{1}=0$. Thus, we need to know the cohomology of $\gamma, H(\gamma)$, for computing the piece of highest antighost number in (50). From the definitions of $\gamma$ acting on the generators of the BRST complex (see the relations (44-48)), we remark that $H(\gamma)$ is generated by

$$
\Phi^{\alpha}=\left(F_{i j}^{a}=\partial_{[i} A_{j]}^{a}, \varphi_{a}, \pi_{a}^{0}, p_{a}^{i}, \pi_{i j}^{a}, \partial_{i} B_{a}^{0 i}\right)
$$


together with their spatial derivatives, by the antighosts 29 30 and their derivatives, as well as by the undifferentiated ghosts $\eta^{(2) a}, \eta_{a}^{i j k}$ and $C_{i j k}^{a}$. Consequently, the general solution to the equation $\gamma a=0$ can be written (up to a trivial term) as

$$
a=a_{M}\left(\left[\Phi^{\alpha}\right],\left[\mathcal{P}_{a_{0}}\right],\left[\mathcal{P}_{a_{1}}\right],\left[\mathcal{P}_{a_{2}}\right]\right) e^{M}\left(\eta^{(2) a}, \eta_{a}^{i j k}, C_{i j k}^{a}\right),
$$

where $e^{M}\left(\eta^{(2) a}, \eta_{a}^{i j k}, C_{i j k}^{a}\right)$ stands for a basis in the space of the polynomials in the ghosts. The notation $f[q]$ signifies that $f$ depends on $q$ and its spatial derivatives up to a finite order. As antigh $\left(\stackrel{(J)}{\omega}_{1}\right)=J$ and $\operatorname{gh}\left(\stackrel{(J)}{\omega}_{1}\right)=1$, it follows that $\operatorname{pgh}\left(\stackrel{(J)}{\omega}_{1}\right)=J+1$. Thus, using (52), it results that the general solution to the equation $\gamma \stackrel{(J)}{\omega}_{1}=0$ is

$$
\stackrel{(J)}{\omega}_{1}=a_{J}\left(\left[\Phi^{\alpha}\right],\left[\mathcal{P}_{a_{0}}\right],\left[\mathcal{P}_{a_{1}}\right],\left[\mathcal{P}_{a_{2}}\right]\right) e^{J+1}\left(\eta^{(2) a}, \eta_{a}^{i j k}, C_{i j k}^{a}\right)
$$

where $\operatorname{antigh}\left(a_{J}\right)=J$.

The equation (49) projected on antighost number $(J-1)$ reads as

$$
\delta \stackrel{(J)}{\omega}_{1}+\gamma \stackrel{(J-1)}{\omega}_{1}=\partial_{i} n^{i}
$$

For the equation (54) to possess solutions (in other words, for $\stackrel{(J-1)}{\omega}{ }_{1}$ to exist), it is necessary that the functions $a_{J}$ belong to $H_{J}(\delta \mid \tilde{d})$, where $H_{J}(\delta \mid \tilde{d})$ means the homological space containing objects of antighost number equal to $J$ that are $\delta$-closed modulo the spatial part of the exterior space-time derivative $\tilde{d}$. Translating the Lagrangian results from [12] at the Hamiltonian level, we have that

$$
H_{J}(\delta \mid \tilde{d})=0, \text { for } J>3,
$$

so we can assume that the development (50) stops after the first four terms

$$
\omega_{1}=\stackrel{(0)}{\omega}_{1}+\stackrel{(1)}{\omega}_{1}+\stackrel{(2)}{\omega}_{1}+\stackrel{(3)}{\omega}_{1}
$$

where $\stackrel{(3)}{\omega}_{1}$ results from $(53)$ with $J=3$. In the meantime, the most general representative of $H_{3}(\delta \mid \tilde{d})$ is given by

$$
a_{3}=k_{i j k}\left(\frac{\delta U}{\delta \varphi_{a}} P_{a}^{i j k}-\frac{\delta^{2} U}{\delta \varphi_{a} \delta \varphi_{b}} P_{a}^{[i} P_{b}^{j k]}-\frac{\delta^{3} U}{\delta \varphi_{a} \delta \varphi_{b} \delta \varphi_{c}} P_{a}^{i} P_{b}^{j} P_{c}^{k}\right)
$$


with $k_{i j k}$ some antisymmetric constants, and $U$ an arbitrary function that depends on $\varphi_{a}$, but not on their derivatives. Now, we can completely determine the last component in (56). On the one hand, the elements of $e^{4}\left(\eta^{(2) a}, \eta_{a}^{i j k}, C_{i j k}^{a}\right)$ can be written in the form

$$
C_{i j k}^{a} \eta^{(2) b}, \eta_{a}^{i j k} \eta_{b}^{i^{\prime} j^{\prime} k^{\prime}}, \eta_{a}^{i j k} \eta^{(2) b} \eta^{(2) c}, \eta^{(2) a} \eta^{(2) b} \eta^{(2) c} \eta^{(2) d}
$$

On the other hand, we ask that the resulting deformations are covariant and independent of the space-time dimension. In view of this, the second and fourth elements in (58) should be discarded as they need a three-dimensional antisymmetric symbol in order to be glued to (57). Then, we obtain that

$$
\begin{aligned}
& \stackrel{(3)}{\omega}_{1}=-\frac{1}{4}\left(\frac{\delta M_{b c}^{a}}{\delta \varphi_{d}} P_{d}^{i j k}-\frac{\delta^{2} M_{b c}^{a}}{\delta \varphi_{d} \delta \varphi_{e}} P_{d}^{(2)[i} P_{e}^{j k]}\right. \\
& \left.-\frac{\delta^{3} M_{b c}^{a}}{\delta \varphi_{d} \delta \varphi_{e} \delta \varphi_{f}} P_{d}^{(2) i} P_{e}^{(2) j} P_{f}^{(2) k}\right) \eta_{a i j k} \eta^{(2) b} \eta^{(2) c}-\left(\frac{\delta W_{a b}}{\delta \varphi_{c}} P_{c}^{i j k}\right. \\
& \left.-\frac{\delta^{2} W_{a b}}{\delta \varphi_{c} \delta \varphi_{d}} P_{c}^{(2)[i} P_{d}^{j k]}-\frac{\delta^{3} W_{a b}}{\delta \varphi_{c} \delta \varphi_{d} \delta \varphi_{e}} P_{c}^{(2) i} P_{d}^{(2) j} P_{e}^{(2) k}\right) C_{i j k}^{a} \eta^{(2) b}
\end{aligned}
$$

where $M_{b c}^{a}$ and $W_{a b}$ depend on $\varphi_{a}$, with $M_{b c}^{a}$ antisymmetric in its lower indices. With $\stackrel{(3)}{\omega}_{1}$ at hand, we pass to determining the piece of antighost number two from the first-order deformation. It obeys the equation (54) with $J=3$, that leads to

$$
\begin{aligned}
& \stackrel{(2)}{\omega}_{1}=\left(\frac{\delta W_{a b}}{\delta \varphi_{c}} P_{c}^{i j}+\frac{\delta^{2} W_{a b}}{\delta \varphi_{c} \delta \varphi_{d}} P_{c}^{(2) i} P_{d}^{(2) j}\right)\left(C_{i j}^{a} \eta^{(2) b}+3 C_{i j k}^{a} A^{b k}\right) \\
& +\frac{1}{2}\left(\frac{\delta M_{b c}^{a}}{\delta \varphi_{d}} P_{d}^{i j}+\frac{\delta^{2} M_{b c}^{a}}{\delta \varphi_{d} \delta \varphi_{e}} P_{d}^{(2) i} P_{e}^{(2) j}\right)\left(\frac{1}{2} \eta_{a i j}^{(2)} \eta^{(2) b} \eta^{(2) c}\right. \\
& \left.+3 \eta_{a i j k} \eta^{(2) b} A^{c k}\right)+\left(M_{b c}^{a} \mathcal{P}_{i j k}^{c}+\frac{\delta M_{b c}^{a}}{\delta \varphi_{d}} P_{d[i}^{(2)} \mathcal{P}_{j k]}^{(2) c}\right) \eta_{a}^{i j k} \eta^{(2) b} \\
& +2\left(\frac{\delta W_{a b}}{\delta \varphi_{c}} P_{c[i}^{(2)} \mathcal{P}_{j k]}^{(2) b}+W_{a b} \mathcal{P}_{i j k}^{b}\right) C^{a i j k} .
\end{aligned}
$$

The equation (49) projected on antighost number one becomes

$$
\delta \stackrel{(2)}{\omega}_{1}+\gamma \stackrel{(1)}{\omega}_{1}=\partial^{i} m_{i}
$$


which further yields

$$
\begin{aligned}
& \stackrel{(1)}{\omega}_{1}=-\frac{\delta W_{a b}}{\delta \varphi_{c}}\left(P_{c}^{(2) j} C_{j}^{(2) a} \eta^{(2) b}+P_{c[i}^{(2)} A_{j]}^{b} C^{a i j}\right)-2 W_{a b} C_{i j}^{a} \mathcal{P}^{(2) b i j} \\
& -\frac{1}{2} \frac{\delta M_{b c}^{a}}{\delta \varphi_{d}}\left(P_{d}^{(2) j} B_{a 0 j} \eta^{(2) b} \eta^{(2) c}+P_{d[i}^{(2)} A_{j]}^{c} \eta_{a}^{(2) i j} \eta^{(2) b}+3 P_{d}^{(2) i} \eta_{a i j k} A^{b j} A^{c k}\right) \\
& -M_{b c}^{a}\left(\eta_{a}^{(2) i j} \eta^{(2) b} \mathcal{P}_{i j}^{(2) c}-\eta_{a}^{i j k} A_{[i}^{b} \mathcal{P}_{j k]}^{(2) c}+\frac{1}{2} \mathcal{P}_{a}^{(2)} \eta^{(2) b} \eta^{(2) c}\right) .
\end{aligned}
$$

Acting in the same manner in relation with the equation that governs the antighost number zero element of $\omega_{1}$

$$
\delta \stackrel{(1)}{\omega}_{1}+\gamma \stackrel{(0)}{\omega}_{1}=\partial^{i} l_{i}
$$

we arrive at the solution

$$
\stackrel{(0)}{\omega}_{1}=-W_{a b}\left(H_{0}^{a} \eta^{(2) b}+C_{i}^{(2) a} A^{b i}\right)-M_{b c}^{a}\left(B_{a}^{0 i} \eta^{(2) b} A_{i}^{c}+\frac{1}{2} \eta_{a}^{(2) i j} A_{i}^{b} A_{j}^{c}\right) .
$$

In consequence, we succeeded in finding the complete form of the first-order deformation of the BRST charge for the model under study.

\subsection{Higher-order deformations}

Next, we investigate the consistency of the first-order deformation, described by the equation (5). In view of this, by direct computation we find that $\left[\Omega_{1}, \Omega_{1}\right]=\int d^{3} x \Delta$, with

$$
\begin{aligned}
& \Delta=K^{a b c} t_{a b c}+K_{d}^{a b c} \frac{\delta t_{a b c}}{\delta \varphi_{d}}+K_{d e}^{a b c} \frac{\delta^{2} t_{a b c}}{\delta \varphi_{d} \delta \varphi_{e}}+K_{d e f}^{a b c} \frac{\delta^{3} t_{a b c}}{\delta \varphi_{d} \delta \varphi_{e} \delta \varphi_{f}} \\
& +U_{d}^{a b c} t_{a b c}^{d}+U_{d e}^{a b c} \frac{\delta t_{a b c}^{d}}{\delta \varphi_{e}}+U_{d e f}^{a b c} \frac{\delta^{2} t_{a b c}^{d}}{\delta \varphi_{e} \delta \varphi_{f}}+U_{d e f g}^{a b c} \frac{\delta^{3} t_{a b c}^{d}}{\delta \varphi_{e} \delta \varphi_{f} \delta \varphi_{g}}
\end{aligned}
$$

where we made the notations

$$
\begin{gathered}
t_{a b c}=W_{d c} M_{a b}^{d}+W_{d a} \frac{\delta W_{b c}}{\delta \varphi_{d}}+W_{d b} \frac{\delta W_{c a}}{\delta \varphi_{d}} \\
t_{a b c}^{d}=W_{e[a} \frac{\delta M_{b c]}^{d}}{\delta \varphi_{e}}+M_{e[a}^{d} M_{b c]}^{e}
\end{gathered}
$$




$$
\begin{gathered}
K^{a b c}=C^{a i j k}\left(\eta^{(2) b} \mathcal{P}_{i j k}^{c}-4 \mathcal{P}_{[i j}^{(2) b} A_{k]}^{c}\right)+H_{0}^{a} \eta^{(2) b} \eta^{(2) c}-2 C_{i}^{(2) a} \eta^{(2) b} A^{c i} \\
+C_{i j}^{a}\left(2 A^{b i} A^{c j}+\eta^{(2) b} \mathcal{P}^{(2) c i j}\right) \\
K_{d}^{a b c}=-2 P_{d[i}^{(2)} A_{j]}^{c} C^{a i j} \eta^{(2) b}-6 C_{i j k}^{a} P_{d}^{(2) i} A^{b j} A^{c k}+\frac{1}{3} C^{a i j k} P_{d[i}^{(2)} \mathcal{P}_{j k]}^{(2) c} \eta^{(2) b} \\
+\left(P_{d}^{(2) i} C_{i}^{(2) a}-C_{i j}^{a} P_{d}^{i j}+P_{d}^{i j k} C_{i j k}^{a}\right) \eta^{(2) b} \eta^{(2) c}+2 C^{a i j k} P_{d[i j} A_{k]}^{c} \eta^{(2) b} \\
K_{d e}^{a b c}=-\left(C_{i j}^{a} P_{d}^{(2) i} P_{e}^{(2) j}+P_{d}^{(2)[i} P_{e}^{j k]} C_{i j k}^{a}\right) \eta^{(2) b} \eta^{(2) c} \\
+6 P_{d}^{(2) i} P_{e}^{(2) j} C_{i j k}^{a} \eta^{(2) b} A^{c k} \\
K_{d e f}^{a b c}=-P_{d}^{(2) i} P_{e}^{(2) j} P_{f}^{(2) k} C_{i j k}^{a} \eta^{(2) b} \eta^{(2) c} \\
+U_{d}^{a b c}=\eta_{d}^{(2) i j} A_{i}^{a} A_{j}^{b} \eta^{(2) c}-\eta_{d}^{i j k} A_{i}^{a} A_{j}^{b} A_{k}^{c}+2 \eta_{d}^{i j k} \eta^{(2) b} \mathcal{P}_{[i j}^{(2) a} A_{k]}^{c} \\
+\left(\frac{1}{3} \mathcal{P}_{d}^{(2)} \eta^{(2) c}-B_{d}^{0 i} A_{i}^{c}+\eta_{d}^{(2) i j} \mathcal{P}_{i j}^{(2) c}+\mathcal{P}_{i j k}^{c} \eta_{d}^{i j k}\right) \eta^{(2) a} \eta^{(2) b} \\
+\frac{3}{2} A^{c i} \eta_{d i j k} P_{e}^{(2) j} P_{f}^{(2) k} \eta^{(2) a} \eta^{(2) b} \\
+3 P_{e i}^{(2)} \eta_{d}^{i j k} \eta^{(2) c} A_{j}^{a} A_{k}^{b} \\
\left.U_{d e f}^{a b c}=-\frac{1}{3 !} \eta_{d}^{(2) i j} P_{e[i}^{(2)} A_{j]}^{c}+\eta_{d}^{i j k} P_{e[i}^{(2)} \mathcal{P}_{j k]}^{(2) c}+\frac{1}{2} \eta_{d}^{i j k} P_{e[i j} A_{k]}^{c}\right) \eta^{(2) a} \eta^{(2) b} \\
U_{d e}^{(2)}=\frac{1}{3 !} \eta_{d i j k} P_{e}^{(2) i} P_{f}^{(2) j} P_{g}^{(2) k} \eta^{(2) a} \eta^{(2) b} \eta^{(2) c} \\
\left(72 B_{d 0 i} P_{e}^{(2) i}-P_{e}^{i j} \eta_{d i j}^{(2)}+P_{e}^{i j k} \eta_{d i j k}\right) \eta^{(2) a} \eta^{(2) b} \eta^{(2) c}
\end{gathered}
$$

The equation (5) requires that $\left[\Omega_{1}, \Omega_{1}\right]$ should be $s$-exact. However, none of the terms in (65) is so, hence $\Delta$ must vanish. This takes place if and only if

$$
t_{a b c}=0, t_{a b c}^{d}=0
$$


The solution to (76) reads as

$$
M_{a b}^{c}=\frac{\delta W_{a b}}{\delta \varphi_{c}},
$$

where, in addition, the antisymmetric functions $W_{a b}$ fulfill the identity

$$
W_{e[a} \frac{\delta W_{b c]}}{\delta \varphi_{e}}=0 .
$$

In consequence, the consistency of the first-order deformation of the BRST charge implies that the functions $W_{a b}$ are antisymmetric and check Jacobi's identity (78) corresponding to an open (nonlinear) algebra. Further, we can take $\Omega_{2}=0$, the remaining higher-order deformation equations being satisfied with the choice

$$
\Omega_{k}=0, k>2 .
$$

This completes the approach to the deformed BRST charge of the free model under discussion.

\section{Deformed BRST-invariant Hamiltonian}

In order to analyse the deformation of the BRST-invariant Hamiltonian (38), we initially solve the equation (9). By direct computation we get that

$$
\begin{aligned}
& {\left[\Omega_{1}, H_{0 \mathrm{~B}}\right]=\int d^{3} x\left(-\left(\frac{\delta W_{a b}}{\delta \varphi_{c}} P_{c}^{i j k}-\frac{\delta^{2} W_{a b}}{\delta \varphi_{c} \delta \varphi_{d}} P_{c}^{(2)[i} P_{d}^{j k]}\right.\right.} \\
& \left.-\frac{\delta^{3} W_{a b}}{\delta \varphi_{c} \delta \varphi_{d} \delta \varphi_{e}} P_{c}^{(2) i} P_{d}^{(2) j} P_{e}^{(2) k}\right) C_{i j k}^{a} \eta^{(1) b}-\frac{1}{2}\left(\frac{\delta M_{b c}^{a}}{\delta \varphi_{d}} P_{d}^{i j k}\right. \\
& \left.-\frac{\delta^{2} M_{b c}^{a}}{\delta \varphi_{d} \delta \varphi_{e}} P_{d}^{(2)[i} P_{e}^{j k]}-\frac{\delta^{3} M_{b c}^{a}}{\delta \varphi_{d} \delta \varphi_{e} \delta \varphi_{f}} P_{d}^{(2) i} P_{e}^{(2) j} P_{f}^{(2) k}\right) \eta_{a i j k} \eta^{(2) b} \eta^{(1) c} \\
& +\left(\frac{\delta W_{a b}}{\delta \varphi_{c}} P_{c}^{i j}+\frac{\delta^{2} W_{a b}}{\delta \varphi_{c} \delta \varphi_{d}} P_{c}^{(2) i} P_{d}^{(2) j}\right)\left(C_{i j}^{a} \eta^{(1) b}+3 C_{i j k}^{a} \partial^{k} A_{0}^{b}\right) \\
& +\frac{1}{2}\left(\frac{\delta M_{b c}^{a}}{\delta \varphi_{d}} P_{d}^{i j}+\frac{\delta^{2} M_{b c}^{a}}{\delta \varphi_{d} \delta \varphi_{e}} P_{d}^{(2) i} P_{e}^{(2) j}\right)\left(\eta_{a i j}^{(2)} \eta^{(2) b} \eta^{(1) c}+\frac{1}{2} \eta_{a i j}^{(1)} \eta^{(2) b} \eta^{(2) c}\right. \\
& \left.+3 \eta_{a i j k} \eta^{(2) b} \partial^{k} A_{0}^{c}+3 \eta_{a i j k} \eta^{(1) b} A^{c k}\right)
\end{aligned}
$$




$$
\begin{aligned}
& +3 \frac{\delta M_{b c}^{a}}{\delta \varphi_{d}} P_{d}^{(2) i} \mathcal{P}^{(2) c j k} \eta_{a i j k} \eta^{(1) b}+M_{b c}^{a}\left(\eta_{a}^{i j k} \eta^{(1) b} \mathcal{P}_{i j k}^{c}-\mathcal{P}^{(2) a} \eta^{(2) b} \eta^{(1) c}\right) \\
& -\frac{\delta W_{a b}}{\delta \varphi_{c}} P_{c}^{(2) i}\left(C_{i}^{(1) a} \eta^{(2) b}+C_{i}^{(2) a} \eta^{(1) b}+2 \partial^{j} A_{0}^{b} C_{i j}^{a}\right) \\
& -\frac{\delta M_{b c}^{a}}{\delta \varphi_{d}} P_{d}^{(2) i}\left(B_{a 0 i} \eta^{(2) b} \eta^{(1) c}+\frac{1}{2} \partial^{j} B_{a i j} \eta^{(2) b} \eta^{(2) c}+A^{c j} \eta_{a i j}^{(2)} \eta^{(1) b}\right. \\
& \left.+A^{c j} \eta_{a i j}^{(1)} \eta^{(2) b}+\partial^{j} A_{0}^{c} \eta_{a i j}^{(2)} \eta^{(2) b}+3 \eta_{a i j k} A^{b j} \partial^{k} A_{0}^{c}\right) \\
& -M_{b c}^{a}\left(\eta_{a}^{(1) i j} \eta^{(2) b} \mathcal{P}_{i j}^{(2) c}+\eta_{a}^{(2) i j} \eta^{(1) b} \mathcal{P}_{i j}^{(2) c}-3 \eta_{a}^{i j k} \partial_{i} A_{0}^{b} \mathcal{P}_{j k}^{(2) c}\right) \\
& -W_{a b}\left(H_{0}^{a} \eta^{(1) b}-\partial^{i} H_{i}^{a} \eta^{(2) b}+C_{i}^{(1) a} A^{b i}+C_{i}^{(2) a} \partial^{i} A_{0}^{b}\right) \\
& -M_{b c}^{a}\left(B_{a}^{0 i} \eta^{(2) b} \partial_{i} A_{0}^{c}+B_{a}^{0 i} \eta^{(1) b} A_{i}^{c}-\partial_{i} B_{a}^{i j} \eta^{(2) b} A_{j}^{c}\right. \\
& \left.\left.+\eta_{a}^{(2) i j} A_{i}^{b} \partial_{j} A_{0}^{c}+\frac{1}{2} \eta_{a}^{(1) i j} A_{i}^{b} A_{j}^{c}\right)\right)
\end{aligned}
$$

which offers us the first-order deformation of the BRST-invariant Hamiltonian as

$$
\begin{aligned}
& h_{1}=-\left(\frac{\delta W_{a b}}{\delta \varphi_{c}} P_{c}^{i j k}-\frac{\delta^{2} W_{a b}}{\delta \varphi_{c} \delta \varphi_{d}} P_{c}^{(2)[i} P_{d}^{j k]}\right. \\
& \left.-\frac{\delta^{3} W_{a b}}{\delta \varphi_{c} \delta \varphi_{d} \delta \varphi_{e}} P_{c}^{(2) i} P_{d}^{(2) j} P_{e}^{(2) k}\right) C_{i j k}^{a} A_{0}^{b} \\
& -\frac{1}{2}\left(\frac{\delta M_{b c}^{a}}{\delta \varphi_{d}} P_{d}^{i j k}-\frac{\delta^{2} M_{b c}^{a}}{\delta \varphi_{d} \delta \varphi_{e}} P_{d}^{(2)[i} P_{e}^{j k]}\right. \\
& \left.-\frac{\delta^{3} M_{b c}^{a}}{\delta \varphi_{d} \delta \varphi_{e} \delta \varphi_{f}} P_{d}^{(2) i} P_{e}^{(2) j} P_{f}^{(2) k}\right) \eta_{a i j k} \eta^{(2) b} A_{0}^{c} \\
& +\left(\frac{\delta W_{a b}}{\delta \varphi_{c}} P_{c}^{i j}+\frac{\delta^{2} W_{a b}}{\delta \varphi_{c} \delta \varphi_{d}} P_{c}^{(2) i} P_{d}^{(2) j}\right) C_{i j}^{a} A_{0}^{b} \\
& +\frac{\delta W_{a b}}{\delta \varphi_{c}} P_{c}^{(2) i}\left(H_{i}^{a} \eta^{(2) b}-C_{i}^{(2) a} A_{0}^{b}\right) \\
& +\frac{1}{2}\left(\frac{\delta M_{b c}^{a}}{\delta \varphi_{d}} P_{d}^{i j}+\frac{\delta^{2} M_{b c}^{a}}{\delta \varphi_{d} \delta \varphi_{e}} P_{d}^{(2) i} P_{e}^{(2) j}\right) \times \\
& \left(\eta_{a i j}^{(2)} \eta^{(2) b} A_{0}^{c}+\frac{1}{2} B_{a i j} \eta^{(2) b} \eta^{(2) c}+3 \eta_{a i j k} A_{0}^{b} A^{c k}\right) \\
& +\frac{\delta M_{b c}^{a}}{\delta \varphi_{d}}\left(P_{d[i}^{(2)} \mathcal{P}_{j k]}^{(2) c} \eta_{a}^{i j k} A_{0}^{b}-P_{d}^{(2) i} B_{a 0 i} \eta^{(2) b} A_{0}^{c}\right.
\end{aligned}
$$




$$
\begin{aligned}
& \left.+\frac{1}{2} P_{d[i}^{(2)} A_{j]}^{c} B_{a}^{i j} \eta^{(2) b}-\frac{1}{2} P_{d[i}^{(2)} A_{j]}^{c} \eta_{a}^{(2) i j} A_{0}^{b}\right) \\
& +M_{b c}^{a}\left(\eta_{a}^{(2) i j} A_{0}^{b} \mathcal{P}_{i j}^{(2) c}+\eta_{a}^{i j k} A_{0}^{b} \mathcal{P}_{i j k}^{c}\right. \\
& \left.-\mathcal{P}_{a}^{(2)} \eta^{(2) b} A_{0}^{c}-B_{a}^{i j} \eta^{(2) b} \mathcal{P}_{i j}^{(2) c}\right) \\
& -W_{a b} H_{\mu}^{a} A^{b \mu}-\frac{1}{2} M_{b c}^{a} B_{a}^{\mu \nu} A_{\mu}^{b} A_{\nu}^{c}
\end{aligned}
$$

In (80 81) and further the functions $M_{b c}^{a}$ are expressed as in (77).

Now, we pass to the equation (10). After some computation, we infer that $\left[\Omega_{1}, H_{1}\right]=\int d^{3} x \bar{\Delta}$, where

$$
\begin{aligned}
& \bar{\Delta}=\bar{K}^{a b c} t_{a b c}+\bar{K}_{d}^{a b c} \frac{\delta t_{a b c}}{\delta \varphi_{d}}+\bar{K}_{d e}^{a b c} \frac{\delta^{2} t_{a b c}}{\delta \varphi_{d} \delta \varphi_{e}}+\bar{K}_{d e f}^{a b c} \frac{\delta^{3} t_{a b c}}{\delta \varphi_{d} \delta \varphi_{e} \delta \varphi_{f}} \\
& +\bar{U}_{d}^{a b c} t_{a b c}^{d}+\bar{U}_{d e}^{a b c} \frac{\delta t_{a b c}^{d}}{\delta \varphi_{e}}+\bar{U}_{d e f}^{a b c} \frac{\delta^{2} t_{a b c}^{d}}{\delta \varphi_{e} \delta \varphi_{f}}+\bar{U}_{d e f g}^{a b c} \frac{\delta^{3} t_{a b c}^{d}}{\delta \varphi_{e} \delta \varphi_{f} \delta \varphi_{g}}
\end{aligned}
$$

where the following notations were employed

$$
\begin{gathered}
\bar{K}^{a b c}=H_{\mu}^{a} \eta^{(2) b} A^{c \mu}-C_{i}^{(2) a} A_{0}^{b} A^{c i}-2\left(C_{i j}^{a} A_{0}^{b} \mathcal{P}^{(2) c i j}-C_{i j k}^{a} A_{0}^{b} \mathcal{P}^{c i j k}\right) \\
\bar{K}_{d}^{a b c}=\left(P_{d}^{(2) i} C_{i}^{(2) a}-P_{d}^{i j} C_{i j}^{a}+P_{d}^{i j k} C_{i j k}^{a}\right) \eta^{(2) b} A_{0}^{c}+\frac{1}{2} P_{d}^{(2) i} H_{i}^{a} \eta^{(2) b} \eta^{(2) c} \\
+\left(C^{a i j k} P_{d[i j} A_{k]}^{c}-C^{a i j} P_{d[i}^{(2)} A_{j]}^{c}+2 P_{d[i}^{(2)} \mathcal{P}_{j k]}^{(2) c} C^{a i j k}\right) A_{0}^{b} \\
\bar{K}_{d e}^{a b c}=P_{d}^{(2) i} P_{e}^{(2) j}\left(3 C_{i j k}^{a} A_{0}^{b} A^{c k}-C_{i j}^{a} \eta^{(2) b} A_{0}^{c}\right)-P_{d}^{(2)[i} P_{e}^{j k]} C_{i j k}^{a} \eta^{(2) b} A_{0}^{c} \\
\bar{K}_{d e f}^{a b c}=-P_{d}^{(2) i} P_{e}^{(2) j} P_{f}^{(2) k} C_{i j k}^{a} \eta^{(2) b} A_{0}^{c} \\
\bar{U}_{d}^{a b c}=\frac{1}{2}\left(\eta_{d}^{(2) i j} A_{i}^{a} A_{j}^{b}+\mathcal{P}_{d}^{(2)} \eta^{(2) a} \eta^{(2) b}+2 \eta_{d}^{i j k}\left(\mathcal{P}_{[i j}^{(2) a} A_{k]}^{b}+\eta^{(2) b} \mathcal{P}_{i j k}^{a}\right)\right) A_{0}^{c} \\
\left(\frac{1}{2} B_{d}^{i j} \eta^{(2) a} \eta^{(2) b}+\eta_{d}^{(2) i j} \eta^{(2) a} A_{0}^{b}\right) \mathcal{P}_{i j}^{(2) c}-\frac{1}{2} B_{d}^{\mu \nu} A_{\mu}^{a} A_{\nu}^{b} \eta^{(2) c} \\
\quad \bar{U}_{d e}^{a b c}=\frac{1}{4}\left(2 P_{e}^{(2) i} B_{d 0 i}-P_{e}^{i j} \eta_{d i j}^{(2)}+P_{e}^{i j k} \eta_{d i j k}\right) \eta^{(2) a} \eta^{(2) b} A_{0}^{c} \\
+\left(\frac{1}{3 !} P_{e i j} \eta^{(2) c}-\frac{1}{4} P_{e[i}^{(2)} A_{j]}^{c}\right) B_{d}^{i j} \eta^{(2) a} \eta^{(2) b}+\eta_{d}^{i j k} P_{e[i}^{(2)} \mathcal{P}_{j k]}^{(2) c} \eta^{(2) a} A_{0}^{b} \\
\quad+\frac{1}{2}\left(P_{e[i}^{(2)} A_{j]}^{b} \eta_{d}^{(2) i j}-P_{e[i j} A_{k]}^{b} \eta_{d}^{i j k}\right) \eta^{(2) a} A_{0}^{c}+\frac{3}{2} P_{e i}^{(2)} \eta_{d}^{i j k} A_{j}^{a} A_{k}^{b} A_{0}^{c}
\end{gathered}
$$




$$
\begin{gathered}
\bar{U}_{d e f}^{a b c}=-\frac{1}{4}\left(P_{e}^{(2) i} P_{f}^{(2) j} \eta_{d i j}^{(2)}+P_{e}^{(2)[i} P_{f}^{j k]} \eta_{d i j k}\right) \eta^{(2) a} \eta^{(2) b} A_{0}^{c} \\
+P_{e}^{(2) i} P_{f}^{(2) j}\left(\frac{1}{3 !} B_{d i j} \eta^{(2) a} \eta^{(2) b} \eta^{(2) c}-\frac{3}{2} \eta_{d i j k} \eta^{(2) a} A^{b k} A_{0}^{c}\right) \\
\bar{U}_{d e f g}^{a b c}=-\frac{1}{4} P_{e}^{(2) i} P_{f}^{(2) j} P_{g}^{(2) k} \eta_{d i j k} \eta^{(2) a} \eta^{(2) b} A_{0}^{c} .
\end{gathered}
$$

Using (76), we find that $\bar{\Delta}=0$, hence we can set $H_{2}=0$. The remaining equations are then satisfied for

$$
H_{k}=0, k>2 .
$$

In this way, the deformed BRST-invariant Hamiltonian is also completely computed.

\section{$6 \quad$ Interacting theory}

At this point, we are in the position to identify the deformed gauge theory corresponding to the starting free model. Putting together the results deduced so far, it follows that the complete expression of the deformed BRST charge consistent to all orders in the deformation parameter is

$$
\begin{aligned}
& \Omega=\int d^{3} x\left(\eta^{(1) a} \pi_{a}^{0}+C_{i}^{(1) a} p_{a}^{i}+\eta_{a}^{(1) i j} \pi_{i j}^{a}-C_{i}^{(2) a}\left(\partial^{i} \varphi_{a}+g W_{a b} A^{b i}\right)\right. \\
& +\eta^{(2) a}\left(-\partial_{i} B_{a}^{0 i}-g \frac{\delta W_{a b}}{\delta \varphi_{c}} B_{c}^{0 i} A_{i}^{b}+g W_{a b} H_{0}^{b}\right) \\
& -\frac{1}{2} \eta_{a}^{(2) i j}\left(\partial_{[i} A_{j]}^{a}+g \frac{\delta W_{b c}}{\delta \varphi_{a}} A_{i}^{b} A_{j}^{c}\right) \\
& +C_{i j}^{a}\left(\partial^{[i} P_{a}^{(2) j]}-g \frac{\delta W_{a b}}{\delta \varphi_{c}} P_{c}^{(2)[i} A^{b j]}-2 g W_{a b} \mathcal{P}^{(2) b i j}\right) \\
& +\eta_{a}^{i j k}\left(\partial_{[i} \mathcal{P}_{j k]}^{(2) a}-g \frac{\delta W_{b c}}{\delta \varphi_{a}} A_{[i}^{c} \mathcal{P}_{j k]}^{(2) b}-\frac{3}{2} g \frac{\delta^{2} W_{b c}}{\delta \varphi_{a} \delta \varphi_{d}} P_{d i}^{(2)} A_{j}^{b} A_{k}^{c}\right) \\
& -g \frac{\delta W_{b c}}{\delta \varphi_{a}}\left(\eta_{a}^{(2) i j} \eta^{(2) b} \mathcal{P}_{i j}^{(2) c}+\frac{1}{2} \mathcal{P}_{a}^{(2)} \eta^{(2) b} \eta^{(2) c}+P_{a}^{(2) i} C_{i}^{(2) b} \eta^{(2) c}\right) \\
& -\frac{g}{2} \frac{\delta^{2} W_{b c}}{\delta \varphi_{a} \delta \varphi_{d}}\left(P_{d}^{(2) i} B_{a 0 i} \eta^{(2) b} \eta^{(2) c}+P_{d[i}^{(2)} A_{j]}^{c} \eta_{a}^{(2) i j} \eta^{(2) b}\right) \\
& +C_{i j k}^{a}\left(\partial^{[i} P_{a}^{j k]}+g \frac{\delta W_{a b}}{\delta \varphi_{c}} A^{b[i} P_{c}^{j k]}+2 g W_{a b} \mathcal{P}^{b i j k}\right.
\end{aligned}
$$




$$
\begin{aligned}
& \left.+3 g \frac{\delta^{2} W_{a b}}{\delta \varphi_{c} \delta \varphi_{d}} P_{c}^{(2) i} P_{d}^{(2) j} A^{b k}+2 g \frac{\delta W_{a b}}{\delta \varphi_{c}} P_{c}^{(2)[i} \mathcal{P}^{(2) b j k]}\right) \\
& +g\left(\frac{\delta W_{b c}}{\delta \varphi_{a}} \mathcal{P}_{i j k}^{c}+\frac{\delta^{2} W_{b c}}{\delta \varphi_{a} \delta \varphi_{d}} P_{d[i}^{(2)} \mathcal{P}_{j k]}^{(2) c}\right) \eta_{a}^{i j k} \eta^{(2) b} \\
& +g\left(\frac{\delta W_{b c}}{\delta \varphi_{a}} P_{a}^{i j}+\frac{\delta^{2} W_{b c}}{\delta \varphi_{a} \delta \varphi_{d}} P_{a}^{(2) i} P_{d}^{(2) j}\right) C_{i j}^{b} \eta^{(2) c} \\
& +\frac{g}{2}\left(\frac{\delta^{2} W_{b c}}{\delta \varphi_{a} \delta \varphi_{d}} P_{d}^{i j}+\frac{\delta^{3} W_{b c}}{\delta \varphi_{a} \delta \varphi_{d} \delta \varphi_{f}} P_{d}^{(2) i} P_{f}^{(2) j}\right)\left(\frac{1}{2} \eta_{a i j}^{(2)} \eta^{(2) b} \eta^{(2) c}\right. \\
& \left.+3 \eta_{a i j k} \eta^{(2) b} A^{c k}\right)-\frac{g}{4}\left(\frac{\delta^{2} W_{b c}}{\delta \varphi_{d} \delta \varphi_{a}} P_{d}^{i j k}-\frac{\delta^{3} W_{b c}}{\delta \varphi_{d} \delta \varphi_{e} \delta \varphi_{a}} P_{d}^{(2)[i} P_{e}^{j k]}\right. \\
& \left.-\frac{\delta^{4} W_{b c}}{\delta \varphi_{d} \delta \varphi_{e} \delta \varphi_{f} \delta \varphi_{a}} P_{d}^{(2) i} P_{e}^{(2) j} P_{f}^{(2) k}\right) \eta_{a i j k} \eta^{(2) b} \eta^{(2) c} \\
& -g\left(\frac{\delta W_{a b}}{\delta \varphi_{c}} P_{c}^{i j k}-\frac{\delta^{2} W_{a b}}{\delta \varphi_{c} \delta \varphi_{d}} P_{c}^{(2)[i} P_{d}^{j k]}\right. \\
& \left.\left.-\frac{\delta^{3} W_{a b}}{\delta \varphi_{c} \delta \varphi_{d} \delta \varphi_{e}} P_{c}^{(2) i} P_{d}^{(2) j} P_{e}^{(2) k}\right) C_{i j k}^{a} \eta^{(2) b}\right)
\end{aligned}
$$

while that of the BRST-invariant Hamiltonian can be written in the form

$$
\begin{aligned}
& H_{\mathrm{B}}=\int d^{3} x\left(-H_{i}^{a}\left(\partial^{i} \varphi_{a}+g W_{a b} A^{b i}\right)\right. \\
& -\frac{1}{2} B_{a}^{i j}\left(\partial_{[i} A_{j]}^{a}+g \frac{\delta W_{b c}}{\delta \varphi_{a}} A_{i}^{b} A_{j}^{c}\right) \\
& +A_{0}^{a}\left(-\partial_{i} B_{a}^{0 i}-g \frac{\delta W_{a b}}{\delta \varphi_{c}} B_{c}^{0 i} A_{i}^{b}+g W_{a b} H_{0}^{b}\right) \\
& +\eta^{(1) a} \mathcal{P}_{a}^{(2)}+C_{i}^{(1) a} P_{a}^{(2) i}+\eta_{a}^{(1) i j} \mathcal{P}_{i j}^{(2) a} \\
& -g \frac{\delta W_{b c}}{\delta \varphi_{a}}\left(\mathcal{P}_{a}^{(2)} \eta^{(2) b} A_{0}^{c}+B_{a}^{i j} \eta^{(2) b} \mathcal{P}_{i j}^{(2) c}-\eta_{a}^{(2) i j} A_{0}^{b} \mathcal{P}_{i j}^{(2) c}\right) \\
& -g \frac{\delta^{2} W_{b c}}{\delta \varphi_{a} \delta \varphi_{d}} P_{d}^{(2) i}\left(B_{a 0 i} \eta^{(2) b} A_{0}^{c}+\eta_{a}^{(2) i j} A_{j}^{c} A_{0}^{b}-B_{a}^{i j} A_{j}^{c} \eta^{(2) b}\right) \\
& +g \frac{\delta W_{a b}}{\delta \varphi_{c}} P_{c}^{(2) i}\left(H_{i}^{a} \eta^{(2) b}-C_{i}^{(2) a} A_{0}^{b}\right) \\
& +g\left(\frac{\delta W_{b c}}{\delta \varphi_{a}} \mathcal{P}_{i j k}^{c}+\frac{\delta^{2} W_{b c}}{\delta \varphi_{a} \delta \varphi_{d}} P_{d[i}^{(2)} \mathcal{P}_{j k]}^{(2) c}\right) \eta_{a}^{i j k} A_{0}^{b}
\end{aligned}
$$




$$
\begin{aligned}
& +\frac{g}{2}\left(\frac{\delta^{2} W_{b c}}{\delta \varphi_{a} \delta \varphi_{d}} P_{d}^{i j}+\frac{\delta^{3} W_{b c}}{\delta \varphi_{a} \delta \varphi_{d} \delta \varphi_{f}} P_{d}^{(2) i} P_{f}^{(2) j}\right) \times \\
& \left(\eta_{a i j}^{(2)} \eta^{(2) b} A_{0}^{c}+\frac{1}{2} B_{a i j} \eta^{(2) b} \eta^{(2) c}+3 \eta_{a i j k} A_{0}^{b} A^{c k}\right) \\
& +g\left(\frac{\delta W_{a b}}{\delta \varphi_{c}} P_{c}^{i j}+\frac{\delta^{2} W_{a b}}{\delta \varphi_{c} \delta \varphi_{d}} P_{c}^{(2) i} P_{d}^{(2) j}\right) C_{i j}^{a} A_{0}^{b} \\
& -\frac{g}{2}\left(\frac{\delta^{2} W_{b c}}{\delta \varphi_{d} \delta \varphi_{a}} P_{d}^{i j k}-\frac{\delta^{3} W_{b c}}{\delta \varphi_{d} \delta \varphi_{e} \delta \varphi_{a}} P_{d}^{(2)[i} P_{e}^{j k]}\right. \\
& \left.-\frac{\delta^{4} W_{b c}}{\delta \varphi_{d} \delta \varphi_{e} \delta \varphi_{f} \delta \varphi_{a}} P_{d}^{(2) i} P_{e}^{(2) j} P_{f}^{(2) k}\right) \eta_{a i j k} \eta^{(2) b} A_{0}^{c} \\
& -g\left(\frac{\delta W_{a b}}{\delta \varphi_{c}} P_{c}^{i j k}-\frac{\delta^{2} W_{a b}}{\delta \varphi_{c} \delta \varphi_{d}} P_{c}^{(2)[i} P_{d}^{j k]}\right. \\
& \left.\left.-\frac{\delta^{3} W_{a b}}{\delta \varphi_{c} \delta \varphi_{d} \delta \varphi_{e}} P_{c}^{(2) i} P_{d}^{(2) j} P_{e}^{(2) k}\right) C_{i j k}^{a} A_{0}^{b}\right) .
\end{aligned}
$$

From the terms of antighost number zero in (92) we see that only the secondary constraints are deformed as

$$
\begin{gathered}
\bar{G}_{a}^{(2)} \equiv-\left(D_{i}\right)_{a}{ }^{b} B_{b}^{0 i}+g W_{a b} H_{0}^{b} \approx 0 \\
\bar{G}_{i j}^{(2) a} \equiv-\frac{1}{2} \bar{F}_{i j}^{a} \approx 0 \\
\bar{\gamma}_{a}^{(2) i} \equiv-D^{i} \varphi_{a} \approx 0
\end{gathered}
$$

where we employed the notations

$$
\begin{gathered}
\left(D_{i}\right)_{a}^{b}=\delta_{a}^{b} \partial_{i}+g \frac{\delta W_{a c}}{\delta \varphi_{b}} A_{i}^{c} \\
\bar{F}_{i j}^{a}=\partial_{[i} A_{j]}^{a}+g \frac{\delta W_{b c}}{\delta \varphi_{a}} A_{i}^{b} A_{j}^{c}, \\
D^{i} \varphi_{a}=\partial^{i} \varphi_{a}+g W_{a b} A^{b i} .
\end{gathered}
$$

The pieces linear in the antighost number one antighosts show that some of the Dirac brackets among the new constraint functions are modified as

$$
\left[\bar{G}_{a}^{(2)}, \bar{G}_{b}^{(2)}\right]=-g\left(\frac{\delta W_{a b}}{\delta \varphi_{c}} \bar{G}_{c}^{(2)}+\frac{\delta^{2} W_{a b}}{\delta \varphi_{c} \delta \varphi_{d}} \bar{\gamma}_{c}^{(2) i} B_{d 0 i}\right)
$$




$$
\begin{gathered}
{\left[\bar{G}_{a}^{(2)}, \bar{G}_{i j}^{(2) b}\right]=g\left(\frac{\delta W_{a c}}{\delta \varphi_{b}} \bar{G}_{i j}^{(2) c}+\frac{1}{2} \frac{\delta^{2} W_{a c}}{\delta \varphi_{b} \delta \varphi_{d}} \bar{\gamma}_{d[i}^{(2)} A_{j]}^{c}\right),} \\
{\left[\bar{G}_{a}^{(2)}, \bar{\gamma}_{b}^{(2) i}\right]=-g \frac{\delta W_{a b}}{\delta \varphi_{c}} \bar{\gamma}_{c}^{(2) i} .}
\end{gathered}
$$

The elements linear in the antighost number two antighosts yield the deformed first-stage reducibility relations under the form

$$
\begin{gathered}
\bar{Z}_{b k l m}^{a i j} \bar{G}_{i j}^{(2) b}+\bar{Z}_{k l m i}^{a b} \bar{\gamma}_{b}^{(2) i}=0, \\
\bar{Z}_{a b}^{i j k l} \bar{G}_{k l}^{(2) b}+\bar{Z}_{a k}^{b i j} \bar{\gamma}_{b}^{(2) k}=0,
\end{gathered}
$$

where the associated first-stage reducibility functions read as

$$
\begin{gathered}
\bar{Z}_{b k l m}^{a i j}=\frac{1}{2}\left(D_{[k}\right)_{b}^{a} \delta_{l}^{i} \delta_{m]}^{j}, \bar{Z}_{k l m i}^{a b}=-\frac{g}{4} \frac{\delta^{2} W_{c d}}{\delta \varphi_{a} \delta \varphi_{b}} g_{i[k} A_{l}^{c} A_{m]}^{d}, \\
\bar{Z}_{a b}^{i j k l}=-g W_{a b}\left(g^{i k} g^{j l}-g^{i l} g^{j k}\right), \bar{Z}_{a k}^{b i j}=\left(D^{[i}\right)_{a}^{b} \delta_{k}^{j]},
\end{gathered}
$$

with

$$
\left(D_{i}\right)^{a}{ }_{b}=\delta^{a}{ }_{b} \partial_{i}-g \frac{\delta W_{b c}}{\delta \varphi_{a}} A_{i}^{c} .
$$

The antighost number three terms underline that the second-stage reducibility relations

$$
\begin{aligned}
& \bar{Z}_{a j_{1} j_{2}}^{b i_{1} i_{2} i_{3}} \bar{Z}_{b k}^{c j_{1} j_{2}} f_{c}^{k}+\bar{Z}_{a b}^{i_{1} i_{2} i_{3} j_{1} j_{2} j_{3}} \bar{Z}_{j_{1} j_{2} j_{3} k}^{b c} f_{c}^{k}= \\
& -g\left(2 \frac{\delta W_{a b}}{\delta \varphi_{c}} \bar{G}^{(2) b\left[i_{1} i_{2}\right.} f_{c}^{\left.i_{3}\right]}+\frac{\delta^{2} W_{a b}}{\delta \varphi_{c} \delta \varphi_{d}} \bar{\gamma}_{c}^{(2)\left[i_{1}\right.} A^{b i_{2}} f_{d}^{\left.i_{3}\right]}\right), \\
& \quad \bar{Z}_{a j_{1} j_{2}}^{b i_{1} i_{2} i_{3}} \bar{Z}_{b c}^{j_{1} j_{2} k l} f_{k l}^{c}+\bar{Z}_{a b}^{i_{1} i_{2} i_{3} j_{1} j_{2} j_{3}} \bar{Z}_{c j_{1} j_{2} j_{3}}^{b k l} f_{k l}^{c}= \\
& \quad 2 g \frac{\delta W_{a b}}{\delta \varphi_{c}} f^{b\left[i_{1} i_{2}\right.} \bar{\gamma}_{c}^{\left.(2) i_{3}\right]},
\end{aligned}
$$

hold on-shell, where $f_{c}^{k}$ and $f_{k l}^{c}$ are arbitrary smooth functions, the latter being antisymmetric in their spatial indices, where the second-stage reducibility functions are given by

$$
\bar{Z}_{a j_{1} j_{2}}^{b i_{1} i_{2} i_{3}}=\frac{1}{2}\left(D^{\left[i_{1}\right.}\right)_{a}^{b} \delta_{j_{1}}^{i_{2}} \delta_{j_{2}}^{\left.i_{3}\right]},
$$




$$
\bar{Z}_{a b}^{i_{1} i_{2} i_{3} j_{1} j_{2} j_{3}}=\frac{g}{3} W_{a b}\left(\sum_{\sigma \in S_{3}}(-)^{\sigma} g^{i_{1} j_{\sigma(1)}} g^{i_{2} j_{\sigma(2)}} g^{i_{3} j_{\sigma(3)}}\right) .
$$

In (111), $S_{3}$ signifies the set of permutations of $\{1,2,3\}$, and $(-)^{\sigma}$ means the parity of the permutation $\sigma$ pertaining to $S_{3}$. Now, we investigate the modified BRST-invariant Hamiltonian (93). The component of antighost number zero

$$
H=\int d^{3} x\left(H_{i}^{a} \bar{\gamma}_{a}^{(2) i}+B_{a}^{i j} \bar{G}_{i j}^{(2) a}+A_{0}^{a} \bar{G}_{a}^{(2)}\right),
$$

represents nothing but the new first-class Hamiltonian, while the terms linear in the antighost number one antighosts give the deformed gauge algebra relations

$$
\begin{gathered}
{\left[H, G_{a}^{(1)}\right]=\bar{G}_{a}^{(2)},} \\
{\left[H, \bar{G}_{a}^{(2)}\right]=g \frac{\delta W_{a b}}{\delta \varphi_{c}}\left(A_{0}^{b} \bar{G}_{c}^{(2)}-\bar{G}_{i j}^{(2) b} B_{c}^{i j}+\bar{\gamma}_{c}^{(2) i} H_{i}^{b}\right)} \\
+g \frac{\delta^{2} W_{a b}}{\delta \varphi_{c} \delta \varphi_{d}}\left(A_{0}^{b} \bar{\gamma}_{c}^{(2) i} B_{d 0 i}-\frac{1}{2} B_{c}^{i j} \bar{\gamma}_{d[i}^{(2)} A_{j]}^{b}\right), \\
{\left[H, G_{i j}^{(1) a}\right]=\bar{G}_{i j}^{(2) a},} \\
{\left[H, \bar{G}_{i j}^{(2) a}\right]=g\left(\frac{\delta W_{b c}}{\delta \varphi_{a}} \bar{G}_{i j}^{(2) c} A_{0}^{b}+\frac{1}{2} \frac{\delta^{2} W_{b c}}{\delta \varphi_{a} \delta \varphi_{d}} A_{0}^{b} \bar{\gamma}_{d[i}^{(2)} A_{j]}^{c}\right),} \\
{\left[H, \gamma_{a}^{(1) i}\right]=\bar{\gamma}_{a}^{(2) i},} \\
{\left[H, \bar{\gamma}_{a}^{(2) i}\right]=g \frac{\delta W_{a b}}{\delta \varphi_{c}} A_{0}^{b} \bar{\gamma}_{c}^{(2) i} .}
\end{gathered}
$$

At this point, we show that the resulting deformations are nontrivial. It is known that trivial deformations can be eliminated by some field redefinitions

$$
z^{A} \rightarrow z^{A}=z^{A}+g \lambda^{A}+O\left(g^{2}\right)
$$

where $\lambda^{A}$ and the higher-order contributions are in general nonlinear functions of $z^{A}$. Now, we invoke the requirement of locality, that plays a key role in quantum field theory. In view of this, we cannot stress enough that the field redefinitions (119) should be local, because otherwise we cannot transform the deformed theory into the initial free one, which essentially are 
both local. Initially, we focus on the deformations of the constraints. Due to the fact that only the secondary constraints are modified, we mainly restrict ourselves to this case. Under the redefinition (119), the first-class constraints (94 96) transform like

$$
\begin{gathered}
\bar{G}_{a}^{(2)} \rightarrow \bar{G}_{a}^{\prime(2)} \equiv-\partial_{i} B_{a}^{0 i}-g \partial_{i}{\stackrel{(B)^{i}}{\lambda}}_{a}+g f_{a}\left(z^{A}\right)+O\left(g^{2}\right) \approx 0, \\
\bar{G}_{i j}^{(2) a} \rightarrow \bar{G}_{i j}^{(2) a} \equiv-\frac{1}{2} \partial_{[i} A_{j]}^{a}-\frac{g}{2} \partial_{[i} \stackrel{(A)}{\lambda}_{j]}^{a}+g f_{i j}^{a}\left(z^{A}\right)+O\left(g^{2}\right) \approx 0, \\
\bar{\gamma}_{a}^{(2) i} \rightarrow \bar{\gamma}_{a}^{\prime(2) i} \equiv-\partial^{i} \varphi_{a}-g \partial^{i} \stackrel{(\varphi)}{\lambda}_{a}+g f_{a}^{i}\left(z^{A}\right)+O\left(g^{2}\right) \approx 0,
\end{gathered}
$$

where the functions denoted by $f$ do not contain derivatives of the fields, and are obtained from (94 96) via (119) (their concrete form can be easily written down, but it is not illuminating in this context). Requiring now that the redefined constraints lead back to those of the free theory, namely, the latter constraints in (13 15), we find at order one in the coupling constant the equations

$$
\begin{gathered}
-\partial_{i} \stackrel{(B)}{\lambda}_{a}^{i}+f_{a}\left(z^{A}\right)=0, \\
-\frac{1}{2} \partial_{[i} \stackrel{(A)}{\lambda}_{j]}^{a}+f_{i j}^{a}\left(z^{A}\right)=0, \\
-\partial^{i} \stackrel{(\varphi)}{\lambda}_{a}+f_{a}^{i}\left(z^{A}\right)=0 .
\end{gathered}
$$

As the functions $f_{a}, f_{i j}^{a}$ and $f_{a}^{i}$ do not involve the derivatives of the fields, it follows that the last equations cannot be satisfied by some local functions $(B)^{i} \quad(A)^{a} \quad(\varphi)$ $\lambda_{a}, \lambda_{i}$ and $\lambda_{a}$. This means that we cannot perform a local transformation of the fields that switches the deformed constraints to their initial form, so the constraints of the interacting theory are indeed nontrivial. Regarding the reducibility functions, it is simple to see that the deformed functions (105 106) and (110-111) reduce to the original ones (25-26) if and only if $W_{a b}\left(\varphi_{a}\right)=0$. However, the last equations cannot be implied by any field redefinition, so the reducibility functions of the coupled model are also nontrivial. Related to the deformed first-class Hamiltonian (112), we observe that it is a combination of deformed first-class constraints, so the result that the modified first-class constraints cannot be brought to their initial form by a local field redefinition then passes on to the first-class Hamiltonian. 
In conclusion, the Hamiltonian deformation of the free model under study results in a nontrivial gauge theory with an open (nonlinear) gauge algebra. As the first-class constraints generate gauge transformations, we expect that the Lagrangian gauge transformations of the resulting theory are modified with respect to the initial ones.

After some computation, we find that the Lagrangian action of the interacting theory is expressed by

$$
S\left[A_{\mu}^{a}, H_{\mu}^{a}, \varphi_{a}, B_{a}^{\mu \nu}\right]=\int d^{4} x\left(H_{\mu}^{a} D^{\mu} \varphi_{a}+\frac{1}{2} B_{a}^{\mu \nu} \bar{F}_{\mu \nu}^{a}\right),
$$

and it is invariant under the gauge transformations

$$
\begin{gathered}
\delta_{\epsilon} A_{\mu}^{a}=\left(D_{\mu}\right)^{a}{ }_{b} \epsilon^{b}, \\
\delta_{\epsilon} H_{\mu}^{a}=\left(D^{\nu}\right)^{a}{ }_{b} \epsilon_{\mu \nu}^{b}-g \frac{\delta W_{b c}}{\delta \varphi_{a}} \epsilon^{b} H_{\mu}^{c} \\
+\frac{g}{2} \frac{\delta^{2} W_{c d}}{\delta \varphi_{a} \delta \varphi_{b}} A^{c \nu} A^{d \rho} \epsilon_{b \mu \nu \rho}+g \frac{\delta^{2} W_{c d}}{\delta \varphi_{a} \delta \varphi_{b}} \epsilon^{c} A^{d \nu} B_{b \mu \nu}, \\
\delta_{\epsilon} \varphi_{a}=-g W_{a b} \epsilon^{b}, \\
\delta_{\epsilon} B_{a}^{\mu \nu}=\left(D_{\rho}\right)_{a}{ }^{b} \epsilon_{b}^{\mu \nu \rho}+g W_{a b} \epsilon^{b \mu \nu}-g \frac{\delta W_{a b}}{\delta \varphi_{c}} \epsilon^{b} B_{c}^{\mu \nu} .
\end{gathered}
$$

As we have anticipated, the deformation of the initial gauge transformations (see (12)) is essentially due to the deformation of the first-class constraints. The gauge transformations of the interacting model are also second-stage reducible, like the original ones, but the reducibility relations take place onshell. We observe that neither the interacting action (126), nor its gauge transformations, contain the four-dimensional antisymmetric symbol, as expected. Moreover, although the gauge structure of the coupled model in four dimensions is richer than that of the one in two dimensions [8], the Lagrangian in (126) has the same expression. This emphasises the possibility to construct nonlinear gauge theories also in dimensions higher than four.

\section{Conclusion}

In conclusion, in this paper we have investigated the consistent Hamiltonian interactions that can be introduced among a set of scalar fields, two types of 
one-forms and a system of two-forms in four dimensions, which are described in the free limit by an abelian BF theory. Our procedure relies on the deformation of both BRST charge and BRST-invariant Hamiltonian of the free version of this model. Related to the deformation of the BRST charge, we find that only its first-order deformation is non-trivial, while its consistency reveals the Jacobi identity for a nonlinear algebra. Concerning the deformation of the BRST-invariant Hamiltonian, we infer again that only its firstorder deformation is non-vanishing. From these two deformed quantities we derive the first-class constraints, accompanying reducibility functions, firstclass Hamiltonian and modified gauge algebra relations of the interacting model, which is precisely a four-dimensional nonlinear gauge theory. This is an example of deformation that modifies the gauge transformations, reducibility relations, as well as the gauge algebra. This result generalizes the two-dimensional analysis exposed in 8$]$ in the sense that although the gauge structure of the four-dimensional model is richer, the Lagrangian of the interacting theory has an expression similar to that from the two-dimensional case. In this light, there is hope that it would be possible to use our deformation procedure in order to construct nonlinear gauge theories in dimensions higher than four.

\section{Acknowledgment}

This work has been supported by a Romanian Council for Academic Scientific Research (CNCSIS) grant.

\section{References}

[1] M. Henneaux, C. Teitelboim, Quantization of Gauge Systems (Princeton University Press, Princeton 1992)

[2] E. S. Fradkin, G. A. Vilkovisky, Phys. Lett. B55 (1975) 224; I. A. Batalin, G. A. Vilkovisky, Phys. Lett. B69 (1977) 309; E. S. Fradkin, T. E. Fradkina, Phys. Lett. B72 (1978) 343; I. A. Batalin, E. S. Fradkin, Phys. Lett. B122 (1983) 157; M. Henneaux, Phys. Rep. 126 (1985) 1

[3] R. Ferraro, M. Henneaux, M. Puchin, J. Math. Phys. 34 (1993) 2757

[4] G. Barnich, Mod. Phys. Lett. A9 (1994) 665 
[5] G. Barnich, M. Henneaux, J. Math. Phys. 37 (1996) 5273

[6] C. Bizdadea, Acta Phys. Polon. B32 (2001) 2843; C. Bizdadea, E. M. Cioroianu, S. O. Saliu, Class. Quantum Grav. 17 (2000) 2007; C. Bizdadea, L. Saliu, S. O. Saliu, Int. J. Mod. Phys. A15 (2000) 893; C. Bizdadea, S. O. Saliu, Phys. Scripta 62 (2000) 261

[7] For a review, see D. Birmingham, M. Blau, M. Rakowski, G. Thompson, Phys. Rep. 209 (1991) 129

[8] C. Bizdadea, Mod. Phys. Lett. A15 (2000) 2047

[9] N. Ikeda, K. I. Izawa, Prog. Theor. Phys. 89 (1993) 223; 1077; 90 (1993) 237; N. Ikeda, Ann. Phys. 235 (1994) 235; P. Schaller, T. Strobl, Phys. Lett. B337 (1994) 266; hep-th/9411163; in Lect. Notes Phys., Vol. 469 (Springer Verlag, Berlin 1996) 321; T. Klösch, P. Schaller, T. Strobl, Helv. Phys. Acta 69 (1996) 305; W. Kummer, H. Liebl, D. V. Vassilevich, Nucl. Phys. B493 (1997) 491

[10] D. J. Gross, A. A. Migdal, Nucl. Phys. B340 (1990) 333; T. Banks, M. O'Loughlin, Nucl. Phys. B362 (1991) 649; E. Witten, Proceedings New York 1991 - Differential Geometry Methods in Theoretical Physics, Vol.1 (World Scientific, Singapore 1991) 176; G. Grignani, G. Nardelli, Class. Quantum Grav. 10 (1993) 2569; T. Fujiwara, Y. Igaraschi, J. Kubo, T. Tabei, Phys. Rev. D48 (1993) 1736; T. Fujiwara, T. Tabei, Y. Igaraschi, K. Maeda, J. Kubo, Mod. Phys. Lett. A8 (1993) 2147; T. Strobl, Int. J. Mod. Phys. D3 (1994) 281; R. Jackiw, gr-qc/9511048

[11] K. Schoutens, A. Sevrin, P. van Nieuwenhuizen, Commun. Math. Phys. 124 (1989) 87; S. Hirano, Y. Kazama, Y. Satoh, Phys. Rev. D48 (1993) 1687; Ö. Dayi, Mod. Phys. Lett. A9 (1994) 2157; Int. J. Mod. Phys. A12 (1997) 4387; D. Louis-Martinez, G. Kunstatter, Phys. Rev. D52 (1995) 3494; M. Blagojević, M. Vasilić, T. Vukašinac, Class. Quantum Grav. 13 (1996) 3003; P. M. Lavrov, P. Y. U. Moshin, Class. Quantum Grav. 16 (1999) 2247; W. Kummer, G. Tieber, Phys. Rev. D59 (1999) 044001; D. Grumiller, D. Hofmann, W. Kummer, Ann. Phys. 290 (2001) 69

[12] G. Barnich, F. Brandt, M. Henneaux, Commun. Math. Phys. 174 (1995) 57; Phys. Rep. 338 (2000) 439 